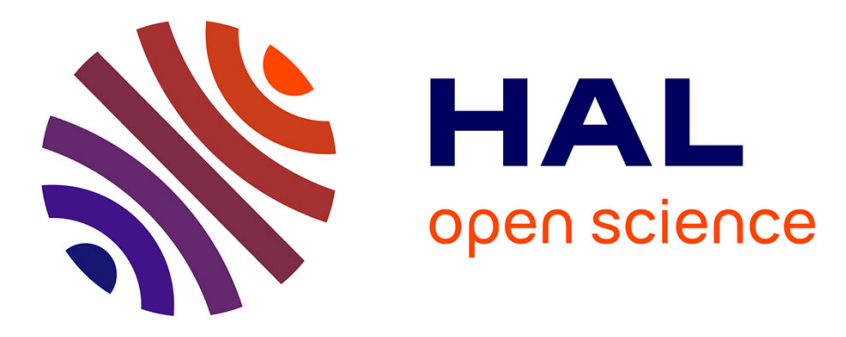

\title{
Opinion dynamics aware marketing strategies in duopolies
}

\author{
Vineeth Varma, Irinel-Constantin Morarescu, Samson Lasaulce, Samuel \\ Martin
}

\section{- To cite this version:}

Vineeth Varma, Irinel-Constantin Morarescu, Samson Lasaulce, Samuel Martin. Opinion dynamics aware marketing strategies in duopolies. 56th IEEE Conference on Decision and Control, CDC 2017, Dec 2017, Melbourne, Australia. pp.3859-3864, 10.1109/cdc.2017.8264227 . hal-01653183v2

\section{HAL Id: hal-01653183 \\ https://hal.science/hal-01653183v2}

Submitted on 30 Nov 2017

HAL is a multi-disciplinary open access archive for the deposit and dissemination of scientific research documents, whether they are published or not. The documents may come from teaching and research institutions in France or abroad, or from public or private research centers.
L'archive ouverte pluridisciplinaire HAL, est destinée au dépôt et à la diffusion de documents scientifiques de niveau recherche, publiés ou non, émanant des établissements d'enseignement et de recherche français ou étrangers, des laboratoires publics ou privés. 


\title{
Opinion Dynamics Aware Marketing Strategies in Duopolies
}

\author{
Vineeth S. Varma, Irinel-Constantin Morărescu, Samson Lasaulce, and Samuel Martin
}

\begin{abstract}
We analyze the competition between two firms when each firm's market share depends on the average opinion of the consumer population about their respective products. All the consumers interact with each other through a social network and these interactions result in a certain dynamics of their opinion. Each firm attempts to sway the public opinion to its own side by spending money on advertising or other marketing tools (like discounts) on specific consumers. We propose a novel model in which the firms are aware of the opinion dynamics and the structure of the social network. As a result, they can prioritize certain consumer nodes over others based on the social graph. We tackle the problem by defining an appropriate static game model and conduct the corresponding equilibrium analysis. Our results are illustrated by a numerical performance analysis which provides several insights into the choice of investment strategies and how they relate to the consumer social network.
\end{abstract}

Keywords: Game theory, Opinion dynamics, Duopoly, Targeted marketing.

\section{INTRODUCTION}

This paper studies how firms will behave, while trying to maximize their revenue by optimizing the advertising or marketing strategy over their consumers in an online social network. These consumers interact with each other over the social network and influence the opinions of each other. Two firms competing for market share from a common consumer pool are analyzed as a non-cooperative duopoly.

In the field of economics and marketing research, noncooperative games between firms associated with advertising costs and the resulting sales or market share have been studied since 1958 [1], [2], [3]. However, these models assume a homogenous population of consumers, and even recent studies on this topic like [4] do not consider that firms have access to data from social networks. Recently [5] investigates the auction mechanisms that are being used by search engines to sell online advertising, i.e., targeted advertising. Our contribution is similar in the sense that it considers targeted advertising by firms. However, unlike [5] we use an opinion dynamics model to propagate the advertising in the social network. Therefore, the firms have to target the "popular/central" users of social networks and try to optimize their advertising strategy in order to maximize their revenue generated by obtaining a higher market share. The key difference is that instead of targeting users based on just their behavior (or opinions), firms are also aware of the

V. S. Varma, I-C. Morărescu and S. Martin are with the Université de Lorraine, CRAN, UMR 7039 and the CNRS, CRAN, UMR 7039, France, \{vineeth. satheeskumar-varma\}@univ-lorraine.fr. S. Lasaulce is with the Laboratoire des Signaux et Systemes (L2S, CNRSCentraleSupelec-Univ. Paris Sud), Gif-sur-Yvette, France. impact popular users can have on the opinion of others. For example, people may discuss over a social network which brand of cola they prefer. We are interested in the marketing strategies of these firms when they optimize their advertising or marketing strategies based on the structure of the social network and the dynamics of opinion, which they obtain from a third party like Google or Facebook.

Opinion dynamics has been studied over the last few decades by researchers from various disciplines such as sociology [6], [7], physics [8], computer science [9], [10], mathematics, and engineering [11], [12], [13]. The primary motivation behind these studies was to better understand the propagation of ideas and fads in social networks. Naturally, some of these opinions that are propagated over a social network will be on products sold by certain firms, which is the framework of interest for this work. Among the many existing models of opinion dynamics, we choose to use an early one proposed in [14]. It considers a fixed network in which individuals repeatedly update their opinion by averaging the opinions of their neighbors. Under some mild assumptions on the network structure, this dynamics always leads to consensus.

In this paper we consider a duopoly in which each firm tries to choose the best marketing strategy based on the information it has on the social network, i.e., each firm can identify the impact of a user's opinion on his neighbors and how this opinion can propagate. In other words, the two firms compete for consumers who belongs to a social network with known topology, and try to shift their opinion to their side, thereby capturing a larger market share.

The contribution of this work is twofold. First, we introduce a new duopoly model which accounts for the fact that the firms know that the consumer's opinion evolves between two consecutive marketing campaigns according to a structured social network. The knowledge about the social network structure can therefore, be exploited to design the marketing strategy. The proposed model allows one to capture and translate mathematically intuitions such as the following. If a firm can convince a very popular user to use its product, this will return in a larger market share when compared to convincing an unpopular user. Traditionally, firms sponsor celebrities to influence their fan-base, however, we are interested in incentives firms can offer to regular, but users with a significant enough influence on other users to capture a larger market share. This is because the popular/influential user can spread his favorable opinion towards the product thought the social network much more than other users. In our work, the scale of investment a firm makes on a user is small, i.e., targeted advertising or 
offering discounts, rather than sponsoring/paying money to a celebrity. The second contribution of the present paper is related to the game-theoretical analysis of the proposed model. We conduct the equilibrium analysis of the proposed game, which includes solving the issues of existence, uniqueness, and determination of a Nash equilibrium (NE).

The rest of the paper is organized as follows. Sec. II formulates the problem under study and introduces the main notation and concepts necessary for the model description. The game-theoretic analysis of the resulting model is presented in Sec. III. In subsection III-A we simplify the model in order to obtain a static game model formulation whose analysis is conducted in subsection III-B. Ideas on how to treat the general and more challenging stochastic game are provided in subsection III-C. Our theoretical results are numerically illustrated in Sec. IV. The paper ends with some concluding remarks and perspectives.

\section{PRoBlem STATEMENT}

We consider a market with Firms 1 and 2 that are interested in attracting consumers to their product. Consumers belong to a social network and we refer to any consumer as an agent. For the sake of simplicity we consider a fixed social network over the set of vertices $\mathcal{V}=\{1,2, \ldots, N\}$ of $N$ agents. In other words, we identify each agent with its index in the set $\mathcal{V}$. To Agent $n \in \mathcal{V}$ we assign a normalized scalar opinion $x_{n}(t) \in[0,1]$ that can be interpreted as the probability of an agent to buy from Firm 1. The revenue obtained by a firm is proportional to its average market share i.e., for Agent $n$ the revenue of Firm 1 is proportional to $x_{n}(t)$ and for Firm 2 the revenue is proportional to $1-x_{n}(t)$. We use $x(t)=\left(x_{1}(t), x_{2}(t), \ldots, x_{N}(t)\right)^{\top}$ to denote the state of the network at any time $t$, where $x(t) \in \mathcal{A}_{0}$ and $\mathcal{A}_{0}=[0,1]^{N}$.

In order to obtain a larger market share, Firm $i$ invests according to the investment or action vector $a_{i}=$ $\left(a_{i, 1}, \ldots, a_{i, N}\right)^{\top} \in \mathcal{A}_{i}$ on marketing campaigns at time instants $t_{j} \in \mathcal{T}$. A given action therefore corresponds to a given marketing campaign, the campaign aiming at influencing the consumer's opinion. These instants corresponding to the campaigns are known and are collected in the set $\mathcal{T}=\left\{t_{1}, t_{2}, \ldots, t_{M}\right\}$ with $t_{M-1}$ being the last campaign. Between two consecutive campaigns, the consumer's opinion is only influenced by the other consumers of the networks.

We call $a_{i}$ the action of Firm $i \in\{1,2\}$, with $a_{i, n} \in\{0,1\}$ being the marketing expenditure targeted at Agent $n$. The action $a_{i}$ of each firm belongs to a discrete set $\mathcal{A}_{1}=\mathcal{A}_{2}=$ $\{0,1\}^{N}$. These campaigns modify the opinions of agents instantaneously according to the function $f\left(a_{0}, a_{1}, a_{2}\right)$ : $\mathcal{A}_{0} \times \mathcal{A}_{1} \times \mathcal{A}_{2} \rightarrow \mathcal{A}_{0}$. We use $\phi\left(a_{0, n}, a_{1, n}, a_{2, n}\right)$ to represent the opinion jump of Agent $n$ such that

$$
f\left(a_{0}, a_{1}, a_{2}\right)=\left(\phi\left(a_{0,1}, a_{1,1}, a_{2,1}\right), \ldots, \phi\left(a_{0, N}, a_{1, N}, a_{2, N}\right)\right)
$$

and we assume that

$$
\phi\left(a_{0, n}, a_{1, n}, a_{2, n}\right)= \begin{cases}a_{0, n} & \text { if } a_{1, n}=a_{2, n} \\ 1 & \text { if } a_{1, n}>a_{2, n} \\ 0 & \text { if } a_{1, n}<a_{2, n}\end{cases}
$$

where $a_{0}$ represents the estimate the firms have about the opinion vector just after the campaign. If the estimate is perfect then $a_{0}=x\left(t_{j}\right), t_{j} \in \mathcal{T}$. The interpretation of this model is that both firms have similar attraction (price or value) to agents when neither or both firms pay for marketing targeted at Agent $n$. In this case, the agent retains his opinion. However, if just one firm invests in $n$, for instance using advertising or offering discounts, this results in the corresponding product being more attractive and the agent therefore shifts his opinion favorably towards that firm.

Let us introduce some graph notions allowing us to define the interaction structure in the social network under consideration.

Definition 1 (Directed graph): A directed graph is a couple $(\mathcal{V}, \mathcal{E})$ with $\mathcal{V}$ being a finite set collecting the vertices, and $\mathcal{E} \subseteq \mathcal{V} \times \mathcal{V}$ the set of directed edges. To each edge $(m, n) \in \mathcal{E}$ we assign a value $A_{m, n}>0$ representing the weight/trust that Agent $m$ gives to Agent $n$. If $(m, n) \in \mathcal{E}$, we say that Agent $m$ is a neighbor of Agent $n$. When $(m, n) \notin \mathcal{E}$ we fix $A_{m, n}=0$. The matrix $\mathbf{A}$ with components $A_{m, n}$ is called the weighted adjacency matrix.

Agent $m$ is said to be connected with Agent $n$ if the graph $(\mathcal{V}, \mathcal{E})$ contains a directed path from $m$ to $n$ i.e., if there exists at least one sequence $\left(m=m_{1}, m_{2}, \ldots, m_{k+1}=n\right)$ such that $\left(m_{h}, m_{h+1}\right) \in \mathcal{E} \Leftrightarrow A_{m_{h}, m_{h+1}}>0, \forall h \in\{1,2, \ldots, k\}$.

Definition 2 (Strongly connected graph): The graph $(\mathcal{V}, \mathcal{E})$ is strongly connected if for any two distinct agents $(m, n) \in \mathcal{V}^{2}, m$ is connected to $n$

In this work we consider that opinion dynamics in the social network is characterized by a linear consensus model described by the following differential equation:

$$
\dot{x}(t)=-\mathbf{L} x(t)
$$

where $\mathbf{L}$ is the Laplacian matrix associated with the graph, whose components are defined as:

$$
L_{m, n}=\left\{\begin{array}{l}
\sum_{n=1}^{N} A_{m, n} \text { if } m=n \\
-A_{m, n} \text { if } m \neq n
\end{array} .\right.
$$

As a result of the marketing campaigns, we have the following hybrid opinion dynamics model

$$
\left\{\begin{array}{llll}
x(t) & =-\mathbf{L} x(t) & & \forall t \in \mathbb{R} \backslash \mathcal{T} \\
x^{+}\left(t_{j}\right) & =f\left(x\left(t_{j}\right), a_{1}, a_{2}\right) & & \forall t_{j} \in \mathcal{T}
\end{array}\right.
$$

as shown in Fig. 3.

For ease of exposition, we first consider the campaign set to be $\mathcal{T}=\{0, T\}$, i.e., the firms launch a marketing campaign at $t=0$ and look at the revenue generated till time $t=\mathrm{T}$. We will later extend to the more general case. As explained previously, the revenue generated by Firm 1 


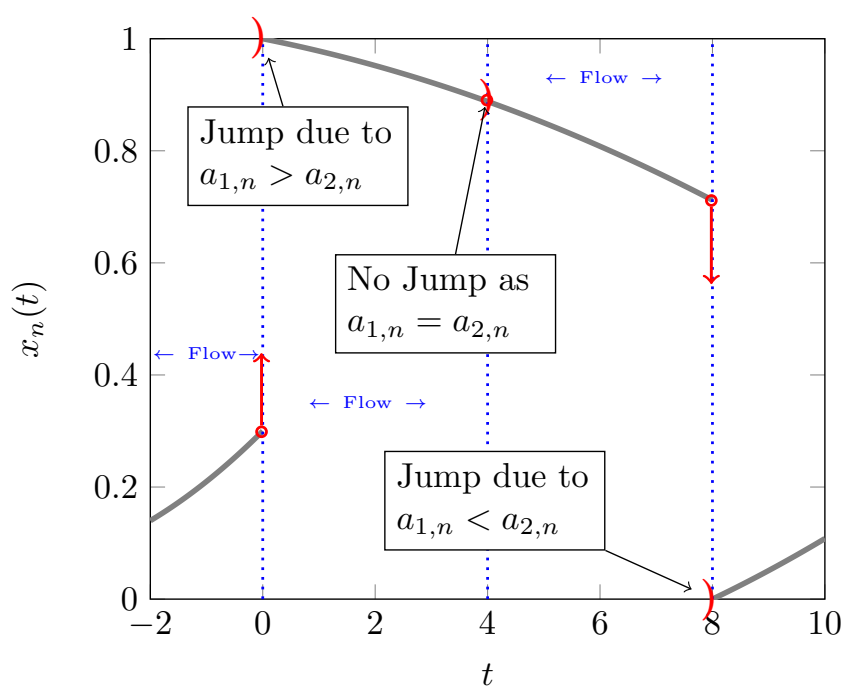

Fig. 1. Each firm tries to influence the consumer's opinion through marketing campaigns which take place at discrete time instants. The two firms conduct their campaigns simultaneous and over a period which is assumed to be negligible. Between two marketing campaigns, the consumer's opinion is only influenced by the interactions it has through the social network it belongs to. The figure shows an example of trajectory for an agent following (5). The trajectory is displayed as a piece-wise continuous lines with jumps when $t \in \mathcal{T}=\{0,4,8,12\}$. The action pairs $\left(a_{1, n}, a_{2, n}\right)$ at $t_{1}=0$ is $(1,0)$, at $t_{2}=4$ is $(1,1)$ and $t_{3}=8$ is $(0,1)$.

over a period $[0, \mathrm{~T}]$ is assumed to be given by

$$
\lambda_{1} \sum_{n \in \mathcal{V}} \int_{0}^{\mathrm{T}} x_{n}(t) \mathrm{d} t
$$

while the revenue generated by Firm 2 is

$$
\lambda_{2} \sum_{n \in \mathcal{V}} \int_{0}^{\mathrm{T}} 1-x_{n}(t) \mathrm{d} t
$$

where $\lambda_{1}, \lambda_{2}>0$ are coefficients associated with the amount of profit earned per agent for unit marketing cost. Due to the expenditure incurred from advertising, the net profit obtained after campaign at time $\mathrm{T}$, which we denote by $u_{i}$ for Firm $i$ can be calculated as

$$
u_{1}\left(a_{0}, a_{1}, a_{2}\right)=\sum_{n \in \mathcal{V}}\left[\left(\lambda_{1} \int_{0}^{\mathrm{T}} x_{n}(t) \mathrm{d} t\right)-a_{1, n}\right],
$$

and

$$
u_{2}\left(a_{0}, a_{1}, a_{2}\right)=\sum_{n \in \mathcal{V}}\left[\left(\lambda_{2} \int_{0}^{\mathrm{T}}\left(1-x_{n}(t)\right) \mathrm{d} t\right)-a_{2, n}\right] .
$$

Although $u_{1}$ and $u_{2}$ in (6) and (7) denote the actual revenue, Firms 1 and 2 may not have access to the actual opinion level of agents $x(0)$ when they launch the advertising campaign. Instead, they might have an estimate of this value which we denote by $\widehat{x}(0)$, which we assume is common to both firms. The study of the case where each firm has a different estimate and incomplete information on the estimate of each other, is beyond the scope of this paper, and is discussed in the conclusion as a potential extension. The dynamics of $x$ will still be given by (5) and the profit computed as (6) and (7) but the actions $a_{1}, a_{2}$ will be decided based on $x(0)=\widehat{x}(0)$ and each firm will estimate its utility using (5) with $x(0)=\widehat{x}(0)$.

\section{GAME-THEORETIC ANALYSIS}

To tackle the above problem in full generality, one would need to study the repeated interactions between the firms and therefore exploit a dynamic game model (a stochastic game to be precise). The choice we make here is to use a static game model to study the problem considered. This allows one to decouple the problem in time (utility functions are independent from stage to stage); some elements on how to move to the stochastic game formulation are provided in Sec. III-C. Note also that the analysis of the static game is a necessary step to the analysis of the stochastic game. To make the static game analysis as simple as possible, we will exploit two auxiliary results. Prop. 1 allows one to express the utility functions (6) and (7) as a function of the initial opinion while Prop. 2 shows that the problem can be decoupled spatially that is, the problem boils down to studying $N$ component games, each component game involving the two firms and one agent.

\section{A. Static game formulation}

As seen in the preceding section, the utility functions depend on the time average opinion. But, by exploiting the agent opinion dynamics (5), each utility function can be shown to depend only on the initial agent opinion and the firm actions. This is what the following proposition states.

Proposition 1: Let us denote by $a_{0}$ the initial opinion vector i.e., $a_{0}:=\widehat{x}(0)$. If $(\mathcal{V}, \mathcal{E})$ is a strongly connected graph then the firm utility functions (6) and (7) can be written as:

$$
u_{1}\left(a_{0}, a_{1}, a_{2}\right)=1_{N}^{\top}\left[\lambda_{1} \mathbf{G} f\left(a_{0}, a_{1}, a_{2}\right)-a_{1}\right]
$$

and

$$
u_{2}\left(a_{0}, a_{1}, a_{2}\right)=1_{N}^{\top}\left\{\lambda_{2}\left[\mathrm{~T} \mathbf{1}_{N}-\mathbf{G} f\left(a_{0}, a_{1}, a_{2}\right)\right]-a_{2}\right\}
$$

where $1_{N}$ is the $N$-dimensional column vector whose entries are all equal to 1 and

$$
\mathbf{G}:=-(\mathbf{L}+\mathbf{J})^{-1}\left[e^{-\mathrm{T}(\mathbf{L}+\mathbf{J})}-\mathbf{I}\right]+\left(1+\mathrm{T}-e^{-\mathrm{T}}\right) \mathbf{J}
$$

with: I being the identity matrix and $\mathbf{J}=1_{N} q^{\top} ; q$ is defined by $q^{\top} \mathbf{L}=0$ and $q^{\top} 1_{N}=1$.

Proof: See Appendix A.

The strategic or normal form of the static game of interest therefore writes as:

$$
\mathcal{G}=\left(\mathcal{F},\left\{\mathcal{A}_{i}\right\}_{i \in \mathcal{F}},\left\{u_{i}\right\}_{i \in \mathcal{F}}\right)
$$

where:

- $\mathcal{F}=\{1,2\}$ is the set of players (i.e., Firms 1 and 2);

- $\mathcal{A}_{i}=\{0,1\}^{N}$ is the set of pure actions for Firm $i$;

- $u_{i}$ is the utility function for Firm $i$ as defined per (8) and (9). 
Now, we introduce a very useful quantity: the agent influential power (AIP). It is useful to derive the proposition on spatial decoupling and represents a key parameter for the numerical performance analysis. The AIP is defined as follows:

$$
\forall n \in\{1, \ldots, N\}, \rho_{n}:=e_{n}^{\top} \mathbf{G}
$$

where $e_{n}$ is the $n^{\text {th }}$ vector of the canonical basis of $\mathbb{R}^{N}$ i.e., the entries are all zero except for the $n^{\text {th }}$ which equals 1 . The AIP appears in the following auxiliary game, which will be referred to as a component game:

$$
\mathcal{G}_{n}=\left(\mathcal{F},\{0,1\}^{2},\left\{u_{i, n}\right\}_{i \in \mathcal{F}}\right)
$$

with

$$
u_{1, n}\left(a_{0, n}, a_{1, n}, a_{2, n}\right)=\lambda_{1} \rho_{n} \phi\left(a_{0, n}, a_{1, n}, a_{2, n}\right)-a_{1, n}
$$

and

$u_{2, n}\left(a_{0, n}, a_{1, n}, a_{2, n}\right)=\lambda_{2} \rho_{n}\left[1-\phi\left(a_{0, n}, a_{1, n}, a_{2, n}\right)\right]-a_{2, n}$

where $\phi$ is defined by (2) and $a_{0, n}=\widehat{x}_{n}(0)$. Therefore, in $\mathcal{G}_{n}$, Firm $i$ only chooses a binary action namely, to invest or not in marketing for Agent $n$, and the impact of the action on the total utility $u_{i}$ is measured by $u_{i, n}$. It is seen that the AIP is a key parameter for the firms since it represents the economic efficiency or the return of investment. In the next section, we show that it suffices to study this component game to analyze the "large" game $\mathcal{G}$.

\section{B. Equilibrium analysis of $\mathcal{G}_{n}$}

In the presence of multiple utility functions for which the players have only partial control of the function variables, it is necessary to define the problem before solving it, hence the notion of the solution concept associated with a game is considered (see e.g., [15]). A famous solution concept is the Nash equilibrium (NE). There exist many interpretations to explain that such an equilibrium may be a reasonable outcome for a problem such as the one considered; this includes learning procedures which only require local or measurable quantities [15]. An NE for $\mathcal{G}$ is defined as follows.

Definition 3 (Pure NE): A strategy profile $\left(a_{1}^{\star}, a_{2}^{\star}\right) \in$ $\{0,1\}^{2 N}$ is a pure NE for $\mathcal{G}$ for a given $a_{0}$ if

$$
\forall i \in \mathcal{F}, \forall a_{i} \in \mathcal{A}_{i}, u_{i}\left(a_{0}, a_{i}^{\star}, a_{-i}^{\star}\right) \geq u_{i}\left(a_{0}, a_{i}, a_{-i}^{\star}\right)
$$

where $-i$ stands for the other firm than $i$.

To conduct the equilibrium analysis of $\mathcal{G}$ we resort to the following auxiliary result.

Proposition 2 (Spatial decoupling): Any pure NE of $\mathcal{G}$ writes as $a^{\star}=\left(a_{1,1}^{\star}, \ldots, a_{1, N}^{\star}, a_{2,1}^{\star}, \ldots, a_{2, N}^{\star}\right)$ where $\forall n \in$ $\{1, \ldots, N\},\left(a_{1, n}^{\star}, a_{2, n}^{\star}\right)$ forms a pure NE for $\mathcal{G}_{n}$.

Proof: See Appendix B.

Based on the previous result, we can now conduct the NE analysis for $\mathcal{G}_{n}$. The main issue of the equilibrium analysis is the existence one. Since the number of players and the number of actions of $\mathcal{G}_{n}$ are finite, the existence of an NE in the sense of the probability distributions is guaranteed [16]; we will come back to this form of NE (called a mixed NE) a little farther into the paper. Here, we start with the existence issue for a pure NE, which is the most relevant type of $\mathrm{NE}$ in practice [15].

Proposition 3 (Equilibrium conditions): Let $\bar{a}_{0, n}=1-$ $a_{0, n}$ and $\mathcal{N}_{n}$ denote the set of pure NE of $\mathcal{G}_{n}$. Then,

1) $(1,1) \in \mathcal{N}_{n}$ iff $\lambda_{1} \geq \frac{1}{\rho_{n} a_{0, n}}$ and $\lambda_{2} \geq \frac{1}{\rho_{n} \bar{a}_{0, n}}$;

2) $(1,0) \in \mathcal{N}_{n}$ iff $\lambda_{1} \geq \frac{1}{\rho_{n} \bar{a}_{0, n}}$ and $\lambda_{2} \leq \frac{1}{\rho_{n} \bar{a}_{0, n}}$;

3) $(0,1) \in \mathcal{N}_{n}$ iff $\lambda_{1} \leq \frac{1}{\rho_{n} a_{0, n}}$ and $\lambda_{2} \geq \frac{1}{\rho_{n} a_{0, n}}$;

4) $(0,0) \in \mathcal{N}_{n}$ iff one of the following conditions is met: (i) $\lambda_{1} \leq \frac{1}{\rho_{n} a_{0, n}}$ and $\lambda_{2} \leq \frac{1}{\rho_{n} \bar{a}_{0, n}}$; (ii) $\lambda_{1} \leq \frac{1}{\rho_{n} \bar{a}_{0, n}}$ and $\lambda_{2} \leq \frac{1}{\rho_{n}} ;$ (iii) $\lambda_{1} \leq \frac{1}{\rho_{n}}$ and $\lambda_{2} \leq \frac{1}{\rho_{n} a_{0, n}}$.

Proof: See Appendix C.

Fig. 2 summarizes Prop. 3 in the $\left(\lambda_{1}, \lambda_{2}\right)$ plane for $a_{0, n}<0.5$. It is worth noting that Prop. 3 emphasizes two extreme situations. When the two firms assigns enough value to the benefit term of their utility namely, when $\lambda_{1}$ and $\lambda_{2}$ are sufficiently large, the firms should invest on the considered agent. Interestingly, the corresponding threshold can be expressed and seen to depend on the API $\rho_{n}$ and the estimate of the agent opinion $a_{0}=\widehat{x}_{0}$. On the other hand, when $\lambda_{1}$ and $\lambda_{2}$ are sufficiently small, the firms have no interest in investing on the considered agent. Prop. 3 also shows the other possible intermediate situations. When none of the conditions of Prop. 3 is satisfied, there is no pure NE. However, there is a strict mixed NE which can be explicitly described. Before expressing it, let us define a mixed NE. A mixed $N E$ is merely a pure $\mathrm{NE}$ of the mixed extension (or expected version) of $\mathcal{G}_{n}$. By denoting $\pi_{i}$ the probability that Firm $i$ assigns to action 1 the expected utility writes as

$$
\widetilde{u}_{i, n}\left(a_{0, n}, \pi_{1}, \pi_{2}\right)=\sum_{k=0}^{1} \sum_{\ell=0}^{1} w_{k, \ell} u_{i, n}\left(a_{0, n}, k, \ell\right)
$$

where $w_{k, \ell}$ is the probability that $\left(a_{1, n}, a_{2, n}\right)=(k, \ell)$, i.e.,

$$
\begin{aligned}
w_{k, \ell}= & k \ell \pi_{1} \pi_{2}+(1-k) \ell\left(1-\pi_{1}\right) \pi_{2} \\
& +(1-\ell) k\left(1-\pi_{2}\right) \pi_{1} \\
& +(1-k)(1-\ell)\left(1-\pi_{1}\right)\left(1-\pi_{2}\right) .
\end{aligned}
$$

Therefore, the profile $\left(\pi_{1}^{\star}, \pi_{2}^{\star}\right)$ is a mixed NE for $\mathcal{G}_{n}$ if it verifies the condition of Definition 3 when replacing $u_{i}$ with $\widetilde{u}_{i}$. The following proposition provides the mixed NE, which is a strictly mixed NE since it lies at the interior of the unit simplices associated with the firm action sets.

Proposition 4: When none of the conditions in Prop. 3 is met, then $\mathcal{G}_{n}$ has a strictly mixed NE that is given by:

$$
\left\{\begin{array}{l}
\pi_{1, n}^{\star}=\frac{1-\lambda_{2} \rho_{n} a_{0, n}}{\left(\lambda_{2} \rho_{n}\right)\left(1-2 a_{0, n}\right)} \\
\pi_{2, n}^{\star}=\frac{1-\lambda_{1} \rho_{n} \bar{a}_{0, n}}{\left(\lambda_{1} \rho_{n}\right)\left(2 a_{0, n}-1\right)} .
\end{array} .\right.
$$

Proof: See Appendix D .

Obviously, while the existence of a mixed NE is appealing mathematically speaking, its operational meaning is not necessarily clear. In fact, as explained in various references 


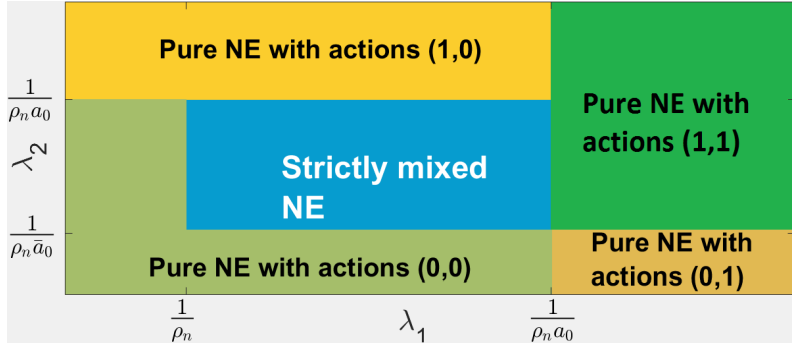

Fig. 2. Conditions for NE shows as a region in the $\left(\lambda_{1} \rho_{n}, \lambda_{2} \rho_{n}\right)$ space for $a_{0, n}=0.25$. The initial opinion of the agents is biased towards Firm 2 , which explains why the above partition is asymmetric.

(see e.g., [17]), at least two operational meanings can be found for a mixed NE. One of them consists in assuming that the interaction is repeated and each firm has to choose the frequency with which it invests on an agent. Another interpretation is given by the Nash mass interpretation, which assumes a large number of agents, or alternately large number of small identical and independent networks. In this situation, the mixed probabilities may represent the fraction of agents for which investing is relevant.

To conclude this section, we consider the special case where the firms have no information about the agent opinion. This case is defined through the following assumption.

Assumption 1: When both firms have no information on the opinion of any agent, both firms take $\widehat{x}_{n}(t)=0.5$ for all $n \in \mathcal{V}$ and $t \in \mathbb{R}$.

Under this assumption, the equilibrium action takes the form given by the following corollary.

Corollary 1: Under Assumption 1 the equilibrium action for Firm $i$ in game $\mathcal{G}_{n}$ writes as

$$
a_{i, n}^{\star}=\left\{\begin{array}{cc}
1 & \text { if } \lambda_{i} \geq \frac{2}{\rho_{n}} \\
0 & \text { if } \lambda_{i}<\frac{2}{\rho_{n}}
\end{array} .\right.
$$

Proof: This can be verified by substituting for $a_{0, n}=$ $\bar{a}_{0, n}=0.5$ in the conditions for NE in Prop. 3 .

Note that when $\lambda_{i} \rho_{n} \neq 2$ for all $(i, n)$, then the game has a unique pure NE. Otherwise it has multiple pure NE. Also, it can be noticed that when $\lambda_{i}<\frac{2}{\rho_{n}}$ for both firms, the game $\mathcal{G}_{n}$ coincides with a prisoner's dilemma, which has been extensively studied in literature [17].

\section{Extension to stochastic games}

As explained at the beginning of Section III, the choice we made is to decouple the opinion dynamics and the dynamics associated with the actions chosen by the firms over time. But, in full generality, the firms interact with each other through a repeated game and over several stages. Therefore, the initial state $a_{0}$ is in fact action-dependent and thus stagedependent. The general model to be considered is therefore a dynamic game in which the game state is controlled by the actions. The full analysis of such a game, especially in the presence of a general observation structure for the estimates available at the firms, is beyond the scope of this paper. Note that, however, the analysis is quite simple for some special cases such as the case with no information about the initial opinions vector. Indeed, under such an information assumption, it is possible to build an investment strategy which relies on the grim trigger strategy [15]. Sufficient conditions e.g., on the discount factor may then be derived to ensure that the corresponding strategy be an equilibrium of the repeated game. This goes beyond the scope of this present work and constitutes a significant extension which may be considered as a future work.

\section{NUMERICAL PERFORMANCE ANALYSIS}

We consider a network of 5 agents interacting with the fixed directed graph shown in Figure 2, yielding heterogeneous $\rho_{n}$. The campaign instances are $\mathcal{T}=\{1,2, \ldots\}$ and we take $T=1$. We consider that Firm 1 is willing to pay more for advertising and he takes $\lambda_{1}=1$ when compared to Firm 2 with $\lambda_{2}=0.5$.

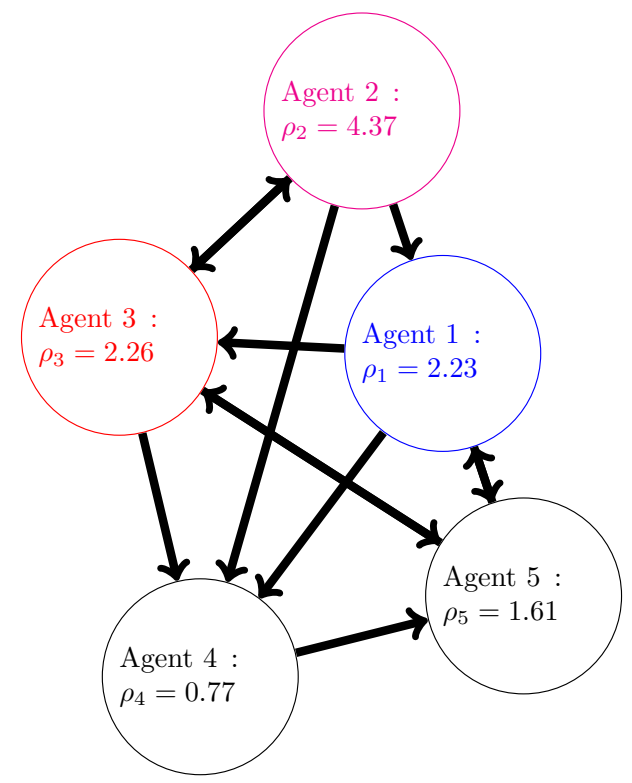

Fig. 3. Graph structure under consideration. An arrow from $m$ to $m$ implies that agent $m$ is influenced by agent $t$ and $A_{n, m}=1$, otherwise $A_{n, m}=0$. We compute $\rho_{n}$ using (12) for $T=1$.

In Figure 4, we plot the opinion against the time when both firms have no information on the opinion following assumption 1. As seen in Figure 4, the aggressive marketing strategy of Firm 1 due to higher $\lambda_{i}$, results in Firm 1 eventually capturing a larger market share. This is because of the existence of the pure NE which is favorable to Firm 1 as $\lambda_{1}>\lambda_{2}$.

In Figure 5, we plot the opinion against the time when both firms are aware of the exact opinion levels $x(t)$. In this case, a pure NE does not always exist, and as a result, firms play a random strategy based on $\rho_{n}$ and $x_{n}(k \mathrm{~T})$ based on (19). What is interesting to note is that although $\lambda_{1}>\lambda_{2}$ as in Figure 4, Firm 1 is unable to capture the market due to the mixed NE. Even running the simulation for a long period 


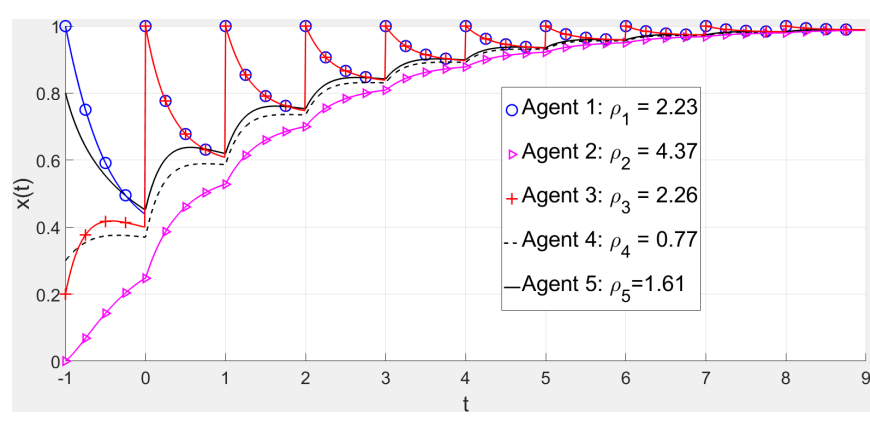

Fig. 4. As both firms have no information, we always have a pure NE, campaign instances are $\mathcal{T}=\{1,2, \ldots\}$. Agent 2 is highly influential and so both firms markets to him, but only Firm 1 markets to 3 and 1 as $\lambda_{1}>\lambda_{2}$. This results in the opinion of 1 and 3 jumping.

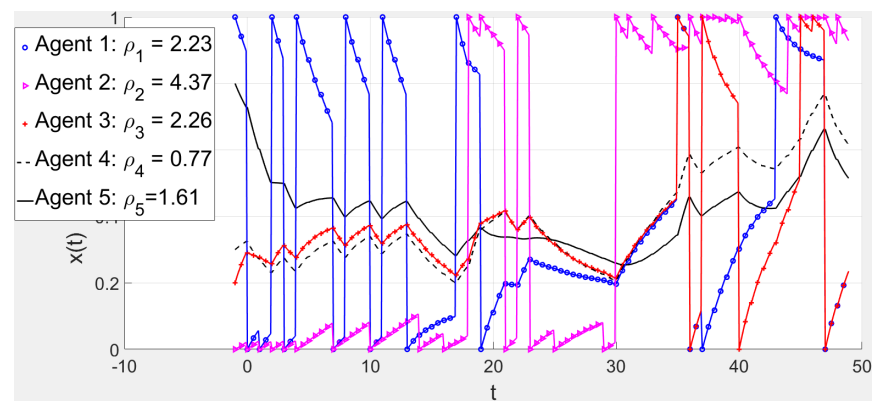

Fig. 5. As both firms have information on $x(t)$, we do not have a pure NE. This results in the opinion of Agents 1,3 , and 2 jumping randomly based on the probabilities in (19) for the mixed NE.

of time does not produce a stable configuration as the firms keep campaigning against each other via a mixed strategy.

Finally, in Figure 6, the average market share captured by firm 1 , normalized to 1 is plotted against $\lambda_{1}$. This value is computed over a long period of time and over several realizations of the mixed strategies. We fix $\lambda_{2}=0.5$ for this figure. What is interesting is that for $\lambda_{1}$ around 1 , the market share capture is actually less than when $\lambda_{1}=0.5$. This implies that even when the firm is more willing to invest in marketing, the NE (pure or mixed) is such that the firms ends up not investing as much as the firm with $\lambda_{2}=0.5$. Nevertheless, when $\lambda_{1}$ is sufficiently high, firm 1 begins to take over the market as expected. In order to further analyze these effects, a highly relevant extension of this work will be when we study the resulting stochastic game.

\section{CONCLUSION}

In this paper, we introduce a novel static game which allows the firms to exploit their knowledge about the social network the targeted consumers belong to. Remarkably, the relationship between the way the marketing campaigns are conducted and the induced revenue for the firms can be well understood. For this, we have conducted a complete equilibrium analysis. Obviously, it is possible to extend the convenient and original model we propose here. Three very significant extensions may be mentioned: 1) To model the problem by a stochastic game to account for the correlations

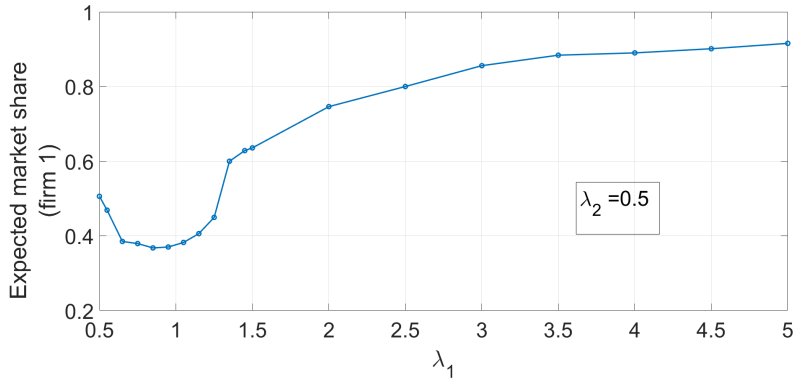

Fig. 6. Average market share captured by firm 1 over a long period of time and over several realizations of the mixed strategies. We fix $\lambda_{2}=0.5$ and plot the average market share (normalized to 1) against $\lambda_{1}$. Surprisingly, increasing $\lambda_{1}$ results in a lower market share initially, and yields returns only when it is sufficiently high.

between the opinions from campaign to campaign. This will give rise to a problem with multiple scale dynamics, that of the interaction among the consumers and that of the interaction between the firms; 2) To study in detail the impact of an arbitrary observation structure on the social network (namely, assume an arbitrary estimate for the social network structure) on the interaction outcome; 3) To conduct a thorough numerical analysis which exploits real data.

\section{APPENDIX}

\section{A. Proof of Prop. 1}

Before proving Prop. 1 we provide below a technical lemma. Recall that $\mathbf{L} \mathbf{1}_{N}=\mathbf{0}_{N}$ and consider the vector $q$ such that $q^{\top} \mathbf{L}=\mathbf{0}_{N}$ with $q^{\top} \mathbf{1}_{N}=1$. Let us also introduce the matrix $\mathbf{J}=\mathbf{1}_{N} q^{\top}$ having the properties $\mathbf{L J}=\mathbf{J L}=$ $\mathbf{0}_{N, N}$ and $\mathbf{J}^{k}=\mathbf{J}, \forall k \in \mathbb{N}$.

Lemma 1: The matrix $\mathbf{L}+\mathbf{J}$ is non-singular.

Proof: Since $(\mathcal{V}, \mathcal{E})$ is strongly connected one has that

$$
\mathbf{L} x=\mathbf{0}_{N} \Leftrightarrow \exists \alpha \in \mathbb{R} \text { such that } x=\alpha \mathbf{1}_{N},
$$

where the value $\alpha$ can be computed as $\alpha=q^{\top} x$. Consequently, one has

$$
P 1: \quad \mathbf{L} x=\mathbf{0}_{N} \text { and } q^{\top} x=0 \text { imply } x=\mathbf{0}_{N} .
$$

Let us now show that

$$
\operatorname{ker}(\mathbf{L}+\mathbf{J})=\mathbf{0}_{N}
$$

which is equivalent with $\mathbf{L}+\mathbf{J}$ non-singular.

Consider $y \in \operatorname{ker}(\mathbf{L}+\mathbf{J})$. It follows that

$$
(\mathbf{L}+\mathbf{J}) y=\mathbf{0}_{N} \Leftrightarrow \mathbf{L} y=-\mathbf{J} y=-\mathbf{1}_{N} q^{\top} y .
$$

Using

$$
q^{\top}(\mathbf{L}+\mathbf{J})=q^{\top} \mathbf{L}+q^{\top} \mathbf{J}=\mathbf{0}_{N}+q^{\top} \mathbf{1}_{N} q^{\top}=q^{\top}
$$

one obtains that $\forall x \in \mathbb{R}^{N}$ the following holds

$$
q^{\top}(\mathbf{L}+\mathbf{J}) x=q^{\top} x .
$$

In particular

$$
q^{\top} y=q^{\top}(\mathbf{L}+\mathbf{J}) y=0 .
$$


Replacing $q^{\top} y$ in (21) one obtains that $\mathbf{L} y=\mathbf{0}_{N}$. We conclude that $y=\mathbf{0}_{N}$ by using $P 1$.

Proof: [Proof of Prop. 1] Let us first observe that (6) and (7) can be rewritten in a more compact form as:

$$
\begin{gathered}
u_{1}\left(a_{0}, a_{1}, a_{2}\right)=\mathbf{1}_{N}^{\top}\left[\lambda_{1} \int_{0}^{\mathrm{T}} x(t) \mathrm{d} t-a_{1}\right] \\
=\mathbf{1}_{N}^{\top}\left[\lambda_{1} \int_{0}^{\mathrm{T}} e^{-\mathbf{L T}} \mathrm{d} t f\left(a_{0}, a_{1}, a_{2}\right)-a_{1}\right] \\
u_{2}\left(a_{0}, a_{1}, a_{2}\right)=\mathbf{1}_{N}^{\top}\left[\lambda_{2} \int_{0}^{\mathrm{T}}\left(\mathbf{1}_{N}-x(t)\right) \mathrm{d} t-a_{1}\right] \\
=\mathbf{1}_{N}^{\top}\left[\lambda_{2}\left(\mathrm{~T} \mathbf{1}_{N}-\int_{0}^{\mathrm{T}} e^{-\mathbf{L T}} \mathrm{d} t f\left(a_{0}, a_{1}, a_{2}\right)\right)-a_{1}\right] .
\end{gathered}
$$

One can see that (8) and (9) are obtained from (22) by replacing $\lambda_{1} \int_{0}^{\mathrm{T}} e^{-\mathbf{L T}} \mathrm{d} t$ with $\mathbf{G}$. To finish the proposition we have to show that $\mathbf{G}$ is given by the expression (10).

Straightforward computation shows that when $\Gamma$ is nonsingular one has

$$
\int_{0}^{\mathrm{T}} e^{\Gamma t} d t=\Gamma^{-1}\left(e^{\Gamma \mathrm{T}}-\mathbf{I}\right)
$$

Consequently, using Lemma 1 we deduce that

$$
\int_{0}^{\mathrm{T}} e^{-(\mathbf{L}+\mathbf{J}) t} d t=-(\mathbf{L}+\mathbf{J})^{-1}\left(e^{-(\mathbf{L}+\mathbf{J}) T}-\mathbf{I}\right) .
$$

On the other hand, as $\mathbf{L J}=\mathbf{J L}$ one has that

$$
e^{-(\mathbf{L}+\mathbf{J}) t}=e^{-\mathbf{L} t} e^{-\mathbf{J} t} .
$$

Using $\mathbf{J}^{k}=\mathbf{J}, \forall k \in \mathbb{N}$ we can express $e^{-\mathbf{J} t}$ as

$$
e^{-\mathbf{J} t}=\mathbf{I}+\mathbf{J} \sum_{k \geq 1} \frac{(-t)^{k}}{k !}=\mathbf{I}+\mathbf{J}\left(e^{-t}-1\right) .
$$

Therefore

$$
e^{-(\mathbf{L}+\mathbf{J}) t}=e^{-\mathbf{L} t}+e^{-\mathbf{L} t} \mathbf{J}\left(e^{-t}-1\right) .
$$

Since $\mathbf{L} \mathbf{J}=\mathbf{0}_{N, N}$ one deduces that $e^{-\mathbf{L} t} \mathbf{J}=\mathbf{J}$ leading to

$$
e^{-(\mathbf{L}+\mathbf{J}) t}=e^{-\mathbf{L} t}+\mathbf{J}\left(e^{-t}-1\right) .
$$

Integrating the previous equality one obtains

$$
\int_{0}^{\mathrm{T}} e^{-\mathbf{L} t} \mathrm{~d} t=\int_{0}^{\mathrm{T}} e^{-(\mathbf{L}+\mathbf{J}) t} d t-\mathbf{J}\left(e^{-\mathrm{T}}-\mathrm{T}-1\right),
$$

and replacing the first term in the right-hand side from (23) we get

$$
\int_{0}^{\mathrm{T}} e^{-\mathbf{L} t} \mathrm{~d} t=-(\mathbf{L}+\mathbf{J})^{-1}\left(e^{-(\mathbf{L}+\mathbf{J}) \mathrm{T}}-\mathbf{I}\right)-\mathbf{J}\left(e^{-\mathrm{T}}-\mathrm{T}-1\right)
$$

which finishes the proof.

\section{B. Proof of Prop. 2}

Proof: Consider any deviating strategy from the concatenated strategy, i.e., consider $a_{i}^{\star}=\left(a_{i, 1}^{\star}, \ldots, a_{i, N}^{\star}\right)$ denoting a concatenated NE of the games $\mathcal{G}_{n}$, and a deviating strategy $a_{i}^{\prime} \notin\left\{\left(a_{i, 1}^{\star}, \ldots, a_{i, N}^{\star}\right)^{\top}\right\}$. By definition of $a_{i, n}^{\star}$ as the NE for $\mathcal{G}_{n}$, we know that if the other firm, i.e., 2 picks $a_{2, n}^{\star}$, then we have

$$
u_{2, n}\left(a_{0, n}, a_{1, n}^{\star}, a_{2, n}^{\prime}\right) \leq u_{2, n}\left(a_{0, n}, a_{1, n}^{\star}, a_{2, n}^{\star}\right)
$$

for any $a_{2}^{\prime} \in \mathcal{A}_{2}$. Now, note that

$$
\begin{aligned}
u_{1}\left(a_{0}, a_{1}, a_{2}\right) & =\mathbf{1}_{N}^{\top}\left[\lambda_{1}\left(\mathbf{G} f\left(a_{0}, a_{1}, a_{2}\right)\right)-a_{1}\right] \\
& =\sum_{n=1}^{N} \rho_{n} \phi\left(a_{0, n}, a_{1, n}, a_{2, n}\right)-a_{1, n} \\
& =\sum_{n} u_{1, n}\left(a_{0, n}, a_{1, n}, a_{2, n}\right)
\end{aligned}
$$

by definition of $\rho_{n}$ in (12) and $u_{1, n}$ in (14). Similarly, we can also express $u_{2}$ as a sum of $u_{2, n}$. From (29) and (26) we can conclude that

$$
u_{2}\left(a_{0}, a_{1}^{\star}, a_{2}^{\prime}\right) \leq u_{2}\left(a_{0}, a_{1}^{\star}, a_{2}^{\star}\right)
$$

for any $a_{2}^{\prime} \in \mathcal{A}_{2}$. With the same reasoning applying for $u_{1}$, we can have that $\left(a_{1}^{\star}, a_{2}^{\star}\right)$ denotes a Nash equilibrium of $\mathcal{G}$. Thus, we have shown that any concatenated NE of $\mathcal{G}_{n}$ is a NE of $\mathcal{G}$. Now, if $a_{i}^{\star}$ is an NE of $\mathcal{G}$, then, consider $a_{i}^{\prime}=a_{i}^{\star}$ but with $a_{i, m} \neq a_{i, m}^{\star}$ for some $m$. By definition of the NE,

$$
u_{1}\left(a_{0}, a_{1}^{\star}, a_{2}^{\star}\right) \geq u_{1}\left(a_{0}, a_{1}^{\prime}, a_{2}^{\star}\right)
$$

for any $a_{i, m}$. Since all the other elements are identical, we can remove them from the inequality to get

$$
u_{1, m}\left(a_{0, m}, a_{1, m}^{\star}, a_{2, m}^{\star}\right) \geq u_{1, m}\left(a_{0, m}, a_{1, m}, a_{2, m}^{\star}\right)
$$

for any $a_{i, m}$, which defines the NE of $\mathcal{G}_{n}$ (similarly for firm 2 ). Therefore any NE of $\mathcal{G}$ automatically has components why are NE of $\mathcal{G}_{n}$.

\section{Proof of Prop. 3}

Proof: For any component game $\mathcal{G}_{n}$, the utility matrix can be written as

$$
\left(\begin{array}{cc}
\lambda_{1} \rho_{n} a_{0, n}, \lambda_{2} \rho_{n} \bar{a}_{0, n} & \lambda_{1} \rho_{n}-1,0 \\
0, \lambda_{2} \rho_{n}-1 & \lambda_{1} \rho_{n} a_{0, n}-1, \lambda_{2} \rho_{n} \bar{a}_{0, n}-1
\end{array}\right)
$$

where the actions corresponding to the the $i$-th row and $j$-th column are $(i-1, j-1)$. We study (30) and search for the conditions when each of the points can be a pure NE.

1) If $\lambda_{1} \rho_{n}-1 \leq \lambda_{1} \rho_{n} a_{0, n}$ and $\lambda_{2} \rho_{n}-1 \leq \lambda_{2} \rho_{n} \bar{a}_{0, n}$, then a resulting pure NE is $(0,0)$.

2) If $\lambda_{1} \rho_{n} a_{0, n} \geq 1$ and $\lambda_{2} \rho_{n} \bar{a}_{0, n} \geq 1$, then a resulting pure NE is $(1,1)$.

3) If $\lambda_{1} \rho_{n} a_{0, n} \leq \lambda_{1} \rho_{n}-1$ and $\lambda_{2} \rho_{n} \bar{a}_{0, n}<1$, then a resulting pure $\mathrm{NE}$ is $(1,0)$.

4) If $\lambda_{2} \rho_{n} \bar{a}_{0, n} \leq \lambda_{2} \rho_{n}-1$ and $\lambda_{1} \rho_{n} a_{0, n} \leq 1$ then a resulting pure $\mathrm{NE}$ is $(0,1)$.

5) Finally, note that when $\lambda_{i} \rho_{n}<1, a_{i, n}^{\star}=0$ as Firm $i$ will never play the action $a_{i, n}=1$. 
When condition 1 is met, the action profile $(0,0)$ is a pure $\mathrm{NE}$ as we see that any agent loses by unilaterally deviating. Similarly, conditions 2-4 ensures that each of the points specified are pure NE. Additionally it can be seen that each of the conditions 1-4 are mutually exclusive, i.e., if 1 is satisfied, then 2-4 can not be satisfied and so on. This can be easily verified (note that $\bar{a}_{0, n}=1-a_{0, n}$ ). Finally, condition 5 can also result in a $(0,0)$ equilibrium, when the other firm does not play 1 , as described in Prop. 3. When none of these conditions are satisfied, none of the points in the payoff matrix correspond to a pure NE, which means that the only NE possible is a mixed equilibrium.

\section{Proof of Prop. 4}

Proof: When none of the conditions in Prop. 3 are met, there are no pure NE for the game. If no point is a pure NE, then a mixed NE must exist, as the game has a discrete action set. This result is shown by Nash in his work [16]. To find this mixed strategy, we use the fact that when player 1 plays at the mixed NE, any action played by player 2 must result in the same expected utility (and vice versa) [17]. Therefore, if $\pi_{1, n}^{\star}$ is the mixed NE strategy of player 1 (the probability of playing 0 ), it must satisfy

$$
\begin{aligned}
\left(1-\pi_{1, n}^{\star}\right) \lambda_{2} \rho_{n} \bar{a}_{0, n}+0 & =\left(1-\pi_{1, n}^{\star}\right)\left(\lambda_{2} \rho_{n}-1\right) \\
& +\pi_{1, n}^{\star}\left(\lambda_{2} \rho_{n} \bar{a}_{0, n}-1\right)
\end{aligned}
$$

which upon simplification gives the first part of (19). We can calculate $\pi_{2, n}^{\star}$ similarly to give the rest of (19).

\section{REFERENCES}

[1] L. Friedman, "Game-theory models in the allocation of advertising expenditures," Operations Research, vol. 6, no. 5, pp. 699-709, 1958.

[2] G. R. Butters, "Equilibrium distributions of sales and advertising prices," The Review of Economic Studies, pp. 465-491, 1977.

[3] X. Vives, "Nash equilibrium with strategic complementarities," Journal of Mathematical Economics, vol. 19, no. 3, pp. 305-321, 1990.

[4] M. Esmaeili, M.-B. Aryanezhad, and P. Zeephongsekul, "A game theory approach in seller-buyer supply chain," European Journal of Operational Research, vol. 195, no. 2, pp. 442-448, 2009.

[5] B. Edelman, M. Ostrovsky, and M. Schwarz, "Internet advertising and the generalized second-price auction: Selling billions of dollars worth of keywords," The American economic review, vol. 97, no. 1, pp. 242259, 2007.

[6] R. Hegselmann and U. Krause, "Opinion dynamics and bounded confidence models, analysis, and simulation," Journal of Artificial Societies and Social Simulation, vol. 5, no. 3, 2002.

[7] A. Martins, "Continuous opinions and discrete actions in opinion dynamics problems," International Journal of Modern Physics C, vol. 19, no. 4, pp. 617-625, 2008.

[8] E. Ising, "Contribution to the theory of ferromagnetism," Ph.D. dissertation, University of Hamburg, 1924.

[9] G. Deffuant, D. Neau, F. Amblard, and G. Weisbuch, "Mixing beliefs among interacting agents," Advances in Complex Systems, vol. 3, pp. 87-98, 2000.

[10] C. V. Kerckhove, S. Martin, P. Gend, P. J. Rentfrow, J. M. Hendrickx, and V. D. Blondel, "Modelling influence and opinion evolution in online collective behaviour," PLOS ONE, vol. 11, no. 6, pp. 1-25, 2016.

[11] E. Yildiz, A. Ozdaglar, D. Acemoglu, A. Saberi, and A. Scaglione, "Binary opinion dynamics with stubborn agents," ACM Transactions on Economics and Computation, vol. 1, no. 4, p. 19, 2013.
[12] I.-C. Morărescu and A. Girard, "Opinion dynamics with decaying confidence: Application to community detection in graphs," IEEE Transactions on Automatic Control, vol. 56, no. 8, pp. 1862 - 1873, 2011.

[13] N. R. Chowdhury, I.-C. Morărescu, S. Martin, and S. Srikant, "Continuous opinions and discrete actions in social networks: a multi-agent system approach," in Proceedings 55th IEEE Conference on Decision and Control, 2016.

[14] M. H. DeGroot, "Reaching a consensus," Journal of the American Statistical Association, vol. 69, no. 345, pp. 118-121, 1974.

[15] S. Lasaulce and H. Tembine, Game Theory and Learning for Wireless Networks : Fundamentals and Applications. Academic Press, 2011.

[16] J. Nash, "Non-cooperative games," Annals of mathematics, pp. 286$295,1951$.

[17] M. Osborne and A. Rubinstein, A Course in Game Theory. The MIT Press, 1994. 